Forum 2021 · 36:122

https://doi.org/10.1007/s12312-021-00912-5

Online publiziert: 26. Februar 2021

(c) Springer Medizin Verlag GmbH, ein Teil von Springer Nature 2021

COVID 19 beschäftigt unser Gesundheitswesen seit einem Jahr und das wird voraussichtlich noch eine ganze Weile so bleiben. Auch in der Krebsversorgung spüren wir den Druck, den die Pandemie auf das Gesundheitssystem ausübt. Zwar werden notwendige Eingriffe und Therapien weiter durchgeführt, doch in einigen Fällen kommt es zu Verschiebungen elektiver Eingriffe und Therapien. Untersuchungsergebnisse, die kürzlich in Oncology Research and Treatment erschienen sind, verdeutlichen das Problem: Nach dem Ende des ersten harten Lockdowns Ende April trat die erwartete Erholung an 75 Krankenhäusern des Betreibers Helios nicht so rasch ein, wie erhofft. Noch Ende Juni verzeichneten die Kliniken 10-20\% weniger Klinikeinweisungen als im gleichen Zeitraum des Vorjahrs. Eine genaue Zuordnung der Gründe lässt sich aus den Daten nicht herleiten. Möglicherweise suchen Menschen bei Beschwerden weniger häufig den Arzt auf oder limitierte Ressourcen in der stationären oder ambulanten Versorgung sind die Ursache. Mit zunehmender Dauer der Pandemie steht zu befürchten, dass der Stau aufgeschobener Untersuchungen und Therapien nachteilige Folgen für die Krebsversorgung unserer Patient*innen hat. Auch die Krebsforschung trifft die Pandemie hart: Die Zahl der Studienstarts in der Onkologie

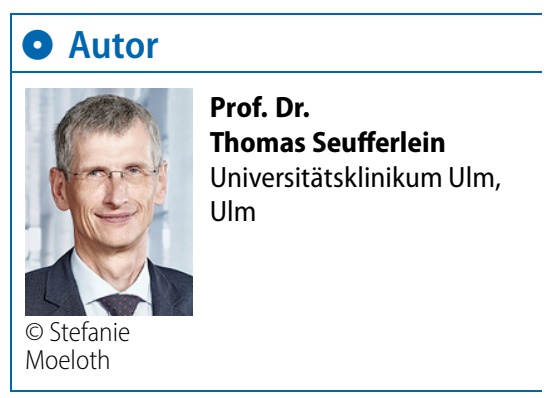

\title{
Thomas Seufferlein
}

Klinik für Innere Medizin I, Universitätsklinikum Ulm, Ulm, Deutschland

\section{COVID-19 und Krebs}

ging in der Zeit von Oktober 2019 bis Mai 2020 im Vergleich zu den Vorjahren um $60 \%$ zurück - das zeigt eine kürzlich in JAMA Network Open publizierte Untersuchung.

Umso erfreulicher, dass Impfstoffe gegen SARS-CoV-2 in einer Rekordzeit entwickelt und zugelassen wurden. Mittlerweile hat das Bundesgesundheitsministerium die erste Fassung der Corona-Impfverordnung überarbeitet. Im Rahmen dieser Anpassungen sind Krebspatient ${ }^{*}$ innen in eine höhere Priorisierungsstufe aufgerückt und haben somit die Chance auf eine frühere Impfung. Auch Angehörige, die Krebsbetroffene zuhause pflegen, haben die Möglichkeit, sich bevorzugt impfen lassen - maximal zwei Angehörige können laut Impfverordnung hier benannt werden. Die Voraussetzung für die Durchsetzung des Anspruchs der Erkrankten ist die Vorlage eines formlosen ärztlichen Attestes oder die Bescheinigung des behandelnden Krankenhauses.

Eine höhere Priorisierung von Krebsbetroffenen ist sicher eine Entwicklung zum Positiven. Als Fachgesellschaft raten wir dringend dazu, dass das für die Impfung nötige Attest oder die Bescheinigung auf der Grundlage einer Beratung mit dem behandlungsführenden Onkologen oder Arzt erstellt wird. Denn nicht immer ist während einer laufenden Krebsbehandlung eine Impfung indiziert; in manchen Fällen nimmt der Impfschutz durch eine onkologische Therapiemaßnahme ab und muss danach aufgefrischt werden. Eine Hilfestellung der DKG mit Empfehlungen zur COVID19-Schutzimpfung bei Krebs zur Unterstützung von Onkologen ist derzeit in Arbeit. $\mathrm{Zu}$ einer angemessenen Impfstrategie für Krebspatient*innen gehört darüber hinaus, dass wir diejenigen op- timal schützen, bei denen eine Impfung aktuell nicht indiziert ist. Nicht nur in stationären, sondern auch in ambulanten Gesundheitseinrichtungen und Praxen muss darauf geachtet werden, dass das Personal, das regelmäßig mit Krebsbetroffenen umgeht, selbst geimpft oder zumindest häufig getestet wird.

Ihr Thomas Seufferlein

Präsident der Deutschen

Krebsgesellschaft

\section{Korrespondenzadresse}

Prof. Dr. Thomas Seufferlein

Klinik für Innere Medizin I, Universitätsklinikum UIm

Albert-Einstein-Allee 23, 89081 Ulm, Deutschland

Thomas.seufferlein@uniklinik-ulm.de

Interessenkonflikt. T. Seufferlein gibt an, dass kein Interessenkonflikt besteht. 